\title{
Flash Flood Detection in Urban Cities Using Ultrasonic and Infrared Sensors
}

\author{
Mustafa Mousa*, Xiangliang Zhang* and Christian Claudel ${ }^{\Psi}$ \\ *King Abdullah University of Science and Technology \\ $\Psi$ The University of Texas at Austin
}

\begin{abstract}
Floods are the most common type of natural disaster. Often leading to loss of lives and properties in the thousands yearly. Among these events, urban flash floods are particularly deadly because of the short timescales on which they occur, and because of the population density of cities. Since most flood casualties are caused by a lack of information on the impending flood (type, location, severity), sensing these events is critical to generate accurate and detailed warnings and short term forecasts. However, no dedicated flash flood sensing systems, that could monitor the propagation of flash floods, in real time, currently exist in cities. In the present paper, firstly a new sensing device that can simultaneously monitor urban flash floods and traffic congestion has been presented. This sensing device is based on the combination of ultrasonic range-finding with remote temperature sensing, and can sense both phenomena with a high degree of accuracy, using a combination of L1-regularized reconstruction and artificial neural networks to process measurement data. Secondly, corresponding algorithms have been implemented on a low-power wireless sensor platform, and their performance in water level estimation in a 6 months test involving four different sensors is illustrated. The results demonstrate that urban water levels can be reliably estimated with error less than $2 \mathrm{~cm}$, and that the preprocessing and machine learning schemes can run in real-time on currently available wireless sensor platforms.
\end{abstract}

Keywords-Water Level Estimation, ARMAX, Nonlinear Regression, Artificial Neural Networks, Flood Detection.

\section{INTRODUCTION}

Wireless sensor networks (WSNs) are widely used for monitoring and control applications [1], [2], [3], [4], [5], [6] such as environmental surveillance [7], [8] or industrial sensing [9], or in the present case, flash flood detection. Floods are one of the most commonly occurring natural disasters [10], accounting for more than half of natural disasters worldwide. They have caused more than 120,000 fatalities in the world between 1991 and 2005 [11], and are a major problem in many areas of the world. While most floods occur outside of urban areas, the recent trend towards urbanization will likely make urban floods more catastrophic due to the concentration of population into relatively small urban areas.

Among floods, flash floods are short fuse weather events, that last less than six hours. Most flood fatalities are in fact caused by flash floods, and most flood victims die because of drowning [12]. This could be avoided by providing accurate flash flood maps to the population in real time. Unfortunately, at the present time little warning exists beyond weather forecasts, which are nonspecific (lack of exact location of the flood, severity of the flood, temporal evolution) and not reliable (i.e. these warnings are associated with a relatively high false alarm rate).

Monitoring floods in real time somehow requires sensing the flooding conditions [13]. Fixed water level sensors are only adapted to river monitoring, and instrumenting entire hydrological basins, which can cover hundreds of square kilometers, is economically infeasible. Satellites are similarly unable to monitor water levels and flows remotely: optical measurements are impossible during floods, and the vertical resolution of current synthetic aperture radars (tens of centimeters) is insufficient for the task.

Existing work ([14], [15], [16], [17]) relies on either contactbased sensors, or non-contact camera-based sensors which are unable to provide a direct water level measurements (such sensors can only provide a binary information: presence of water or not). In [18], the authors use ultrasonic rangefinders to monitor floods, but do not consider the environmental perturbations to the measurement, which is the focus of the present article. Such perturbations severely affect the accuracy of the sensor, and can lead to false or missed detections.

In this article, we propose a new type of flash flood sensor combining ultrasonic rangefinders with passive infrared temperature sensors. This sensor can be used as a backbone for an urban flash flood wireless sensor network architecture, since it can monitor pluviometry, water presence and water level with relatively high accuracy. While the measurement of distances using a calibrated ultrasonic rangefinder is easy, when environmental conditions are well known, the present problem involves the estimation of a distance from time-offlight measurements and from a model of the atmospheric layer between the sensor and the ground. Since this model is encoded by a Partial Differential Equation (PDE) which has numerous uncertain parameters, we choose a non-model based approach to compute the water levels directly, from raw temperature and distance measurements.

The emphasis of the present work is on water level detection (using the proposed sensor [19]), though this sensor can also be simultaneously used for traffic flow monitoring (by monitoring the temperature and distance of disturbances created by vehicles passing by the sensor), or rain rate detection, making it a cost-effective solution. We show that simple temperature correction methods fail to provide a sufficiently accurate measurement, and that machine learning or nonlinear dynamical model-based regression methods can provide a solution to the sensing problem. 
In particular, Artificial Neural Networks (ANN) have an excellent accuracy, and have a low computational complexity (for the chosen number of neurons and layers) which makes it suitable to low-power embedded platforms.

The following list summarizes the contributions of the present article over existing work:

- Novel, dual use (traffic and flash flood) sensor system that addresses a key economic requirement in flash flood sensor networks (since flash floods happen very infrequently).

- New preprocessing scheme based on $L_{1}$ regularization for real-time sensor fault detection and corresponding missing data inference.

- Use of multiple machine learning techniques, ranging from artificial neural networks and fuzzy logic to nonlinear regression on preprocessed sensor measurement data to estimate water levels and learn the proper compensation to apply over all environmental conditions.

- Implementation of the corresponding algorithms on a low-power experimental hardware platform.

- Extensive validation over a period of one year, including the successful detection of the only flash flood event occurring over the period, without any false detection.

The rest of this article is organized as follows. Section II describes the dual ultrasonic/passive infrared sensor network used in this study. Section III describes the nature of the urban flood detection problem and the research questions addressed in this paper, going through the possible models that can be used to estimate water levels using raw measurement data, (include linear models (ARMAX)) and then in the same section, we emphasize on the need of a dedicated preprocessing stage. We then show in section IV our proposed solution (using our sensor nodes described in section II) and its performance comparing it to the existing models using norm two $\left(L_{2}\right)$ and norm infinity $\left(L_{\infty}\right)$ approaches, as well as, investigating the spatio-tempral robustness of the proposed solution model parameters. Section IV-G investigates the detection of an actual minor flooding incident using the proposed approach.

We then describe a computational platform that can solve the proposed neural network training and prediction problems in real time in section $\mathrm{V}$.

\section{SENSING PRINCIPLE}

\section{A. Sensor design considerations}

Sensing flash floods in cities is challenging, since the sensors must have an extended lifetime, measure the water levels in all flow conditions, and be capable of self-monitoring (to make sure they are always functional).

Sensors have been investigated in the past for flood monitoring applications [20], in particular ultrasonic water level measurements on bridges [21], [22] or pressure sensors for water level measurements of rivers [23]. In the present case, the constraints detailed above prevent contact sensors such as pressure transducers [24] from being used. Indeed, these sensors would be unable to measure static and dynamic pressures independently, and their measurements would be affected by their orientation with respect to the water flow. They could also be affected by debris or rocks carried by flash floods, and would need to be periodically tested in water.

Among non-contact sensing technologies, three main technologies could be thought of: ultrasonic rangefinders and Ultra Wide Band (UWB) radars and LIDARs. Ultrasonic rangefinder are much cheaper and more accurate than both UWB and currently available LIDARs, though they can be affected by environmental parameters, such as temperature or humidity. In this article, we choose ultrasonic rangefinders for their very low cost.

\section{B. Sensor description}

The flood sensor node that we investigate in this article [25] contains six passive infrared (PIR) sensors and one ultrasonic (US) rangefinder connected to a microcontroller platform that we have developed for this specific purpose. The six PIR sensors (which are also used for traffic monitoring) are Melexis MLX90614 connected to the platform via SMBus. These sensors measure both the ground temperature in their field of view and their actual temperature. The ultrasonic rangefinder is a MaxBotix MB7066 measuring its distance to objects below it and sending these measurements to a microcontroller via a serial port. The microcontroller platform is developed by our group [26], and is based on an ARM Cortex M4 processor operating at $32 \mathrm{MHz}$.

At this stage, the microcontroller platform applies a median filter to the raw distance and temperature measurements provided by all sensors. The time window of this median filter is currently 30s, and gives a reliable estimate of the actual ground and sensor temperatures, as well as raw ground distance. Median filtering was chosen over moving average filtering because of the presence of vehicular traffic, which creates perturbations in temperature and distance measurements. When traffic is relatively light (which is always the case on the deployment sites), these perturbations are completely cancelled by the median filter. The measurements are sent wirelessly to a sink node, and are then pushed to an input database. The complete system is represented in Figure 1.

The complete sensor structure is made of a lightweight aluminum alloy and weights less than $6 \mathrm{~kg}$. The structure has been designed by our group using CAD tools, and manufactured by a subcontractor. Four of these sensors have been deployed on street lights within two residential areas located $120 \mathrm{~km}$ apart for the dual use of traffic and flash flood monitoring. These sensors are operational since November 29, 2013, and are illustrated in Figure 2.

\section{PROBLEM DEFINITION}

The temperature and distance to the ground measurements generated by the sensor over 6 days (under normal conditions, so that fluctuations are only due to temperature change) are shown in Figure 3. As can be seen from this figure, the raw distance measurements vary significantly over the period (about $12 \mathrm{~cm}$ ) despite the fact that no flooding occurred over this period. These variations in observed distance are caused by the dependency of the speed of sound on air temperature. For most distance measurement applications, the air temperature 


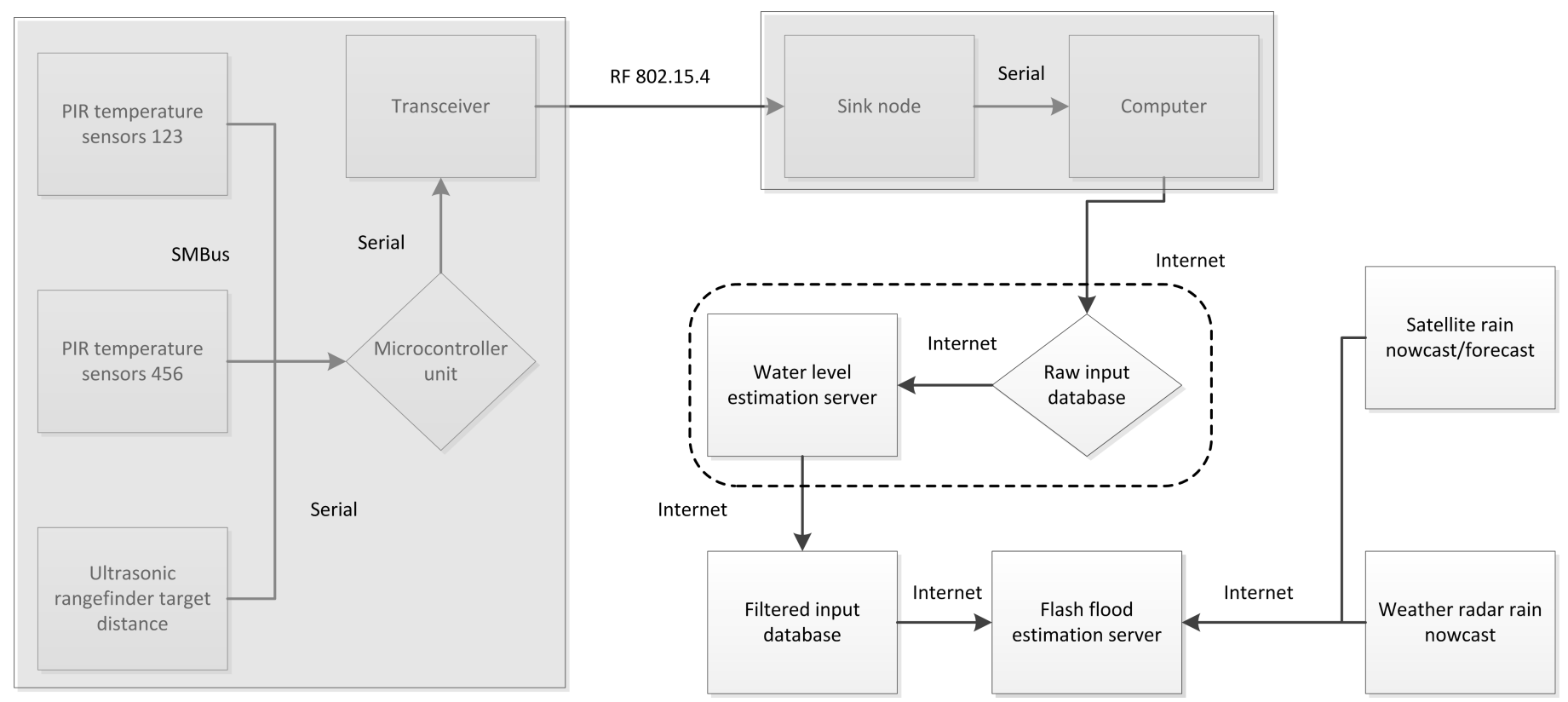

Figure 1: System representation. The sensor described in this article corresponds to the leftmost part of this diagram.

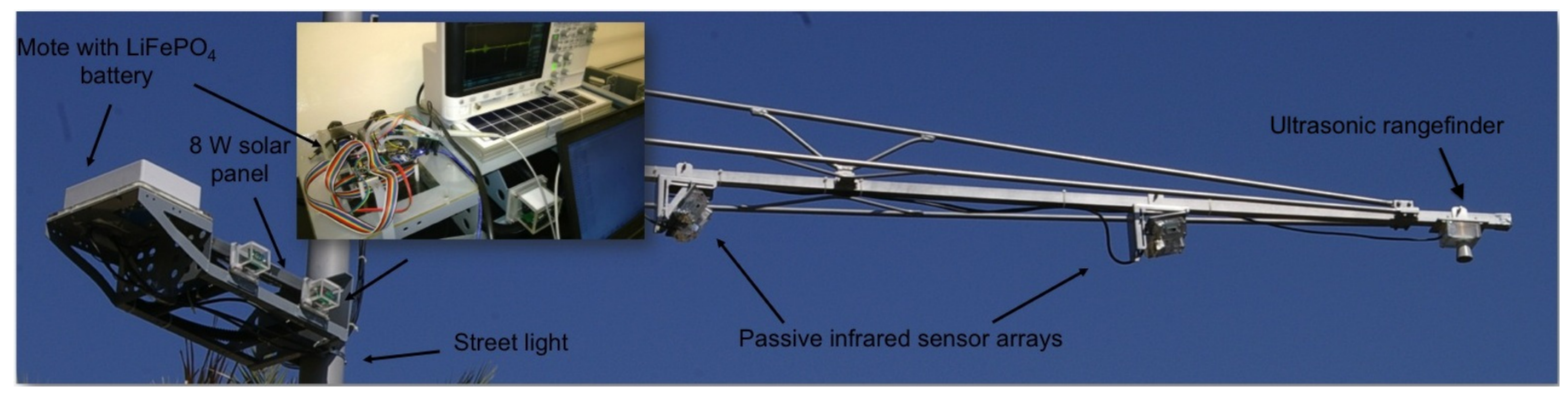

Figure 2: Flood sensor node installed on a public street light in KAUST university campus

effects cause an error that is usually negligible, especially when the temperature of the air is uniform in space. For our sensor, installed at a height of 5 meters (to clear the below traffic) and at usual temperatures, a $1^{\circ} \mathrm{C}$ increase in temperature causes a $1 \mathrm{~cm}$ increase in the measured distance. In addition, the temperature of the air is far from being uniform, with ground temperatures up to $20^{\circ} \mathrm{C}$ higher than air temperatures. Since the distance measurement errors caused by temperature variations are larger than the expected precision of the sensor, we need to somehow estimate the true distance to the ground using the raw distance and temperature measurement data.

This problem can be formulated as follows. The ultrasonic rangefinder measures a time-of-flight (which is converted into an estimated distance by assuming a fixed speed of sound). The time of flight $F(t)$ of the ultrasonic wave (measured at a given time $t$ ) can be computed as follows [27]:

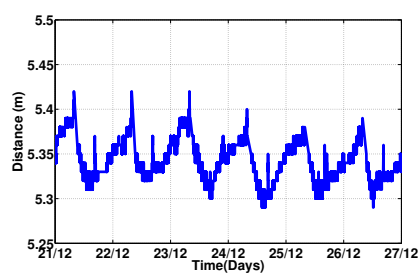

(a)

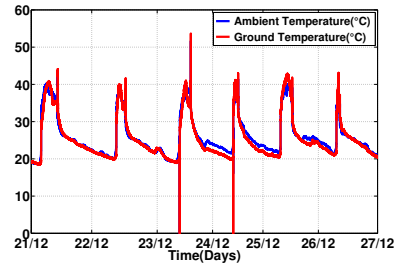

(b)
Figure 3: (a) Raw distance measurements from ultrasound sensor. (b) Ambient and ground temperature measurements.

$$
F(t)=2 \int_{0}^{z_{0}} \frac{d z}{c(\theta(z, t))}
$$


where $\theta(z)$ is the air temperature at altitude $z$. One of the main difficulties arising in this problem is the estimation of the function $\theta(z, t)$. This function depends on multiple factors that are not directly measured by the sensor, including for example cloud coverage, presence of shadow from buildings, heat island effects and ground albedo. To increase the accuracy of the sensor, we need to estimate the correction to apply to the raw distance measurements of the ultrasonic sensor. The correction factor represents all the perturbations caused by the uneven temperature profile in the air layer below it. For the reasons mentioned above, it is infeasible in practice to model the air layer temperature using a PDE, since the boundary conditions and the model parameters of the problem are unknown. We thus investigate several model and nonmodel based approaches to correct the measurements, ranging from linear ARMAX models to ANNs.

In this context, our problem is formalized as follows. Let us denote, by $e(t)$, the difference between the measured (i.e. the raw output of the ultrasonic rangefinder) and the actual ground distance, which is known when no flash flood occurs. Our objective is to estimate $e(t)$ given $T_{a}(\cdot)$ and $T_{g}(\cdot)$, where $T_{a}(\cdot)$ and $T_{g}(\cdot)$ are the sensor measurements of the ambient and ground temperatures respectively.

\section{A. Naive temperature correction}

We first investigate the need for a complex temperature compensation model [28] by checking if simpler naive correction methods can apply. We know that the measured time of flight is given by equation 1 , in which $\theta(z, t)$ is unknown. Let us first assume that $\theta(\cdot, t)$ is constant, that is, the temperature in the air column is uniform. With this assumption, equation 1, becomes:

$$
F(t)=2 \frac{L}{c(\theta(t))}
$$

where $\theta(t)$ is the uniform temperature in the air column. For the two applications below, we used $c(\theta(t))=331.3+0.6$. $\theta(t)$ in $m / s$, which represents the approximate speed of sound in function of the air temperature $\theta(t)$ in ${ }^{\circ} C$, on a narrow temperature range around $20^{\circ} \mathrm{C}$.

In Figure 4, we assume that $\theta(t)=T_{a}(t)$, i.e., the air temperature in the column is identical to the ambient temperature measured by the infrared sensor. This assumes that the heating effects of the sun on the sensors are negligible (that is, the sensor is in thermal equilibrium with the ambient air). This occurs in practice when the wind is very high (which increases the heat transfer between the sensor and the air), or when the solar forcing is negligible (for instance during the night, or during overcast conditions).

As can be seen from Figure 4, the estimation during the night is very good, though the compensation fails during the day. This is caused both by the assumption that the air temperature is uniform (which is wrong during the day), and by the fact that the ambient temperature measured by the sensor can be higher than the air temperature due to solar forcing. To remove the solar forcing effect on the ambient temperature measurement of the PIR sensor, we used calibrated air temperature measurements from the closest weather station. The

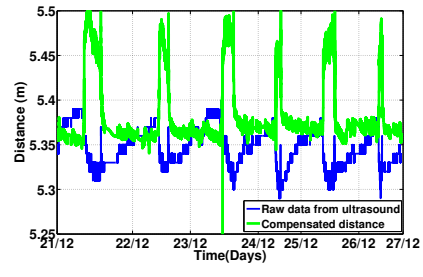

(a)

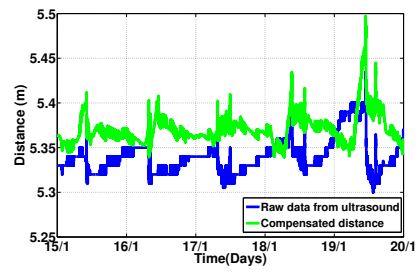

(b)
Figure 4: (a) Predicted distance measurement computed using ambient temperature sensor measurements, and (b) weather station air temperature measurements.

corresponding predicted distance measurements are illustrated in Figure 4, and similarly show large variations with time, whereas the actual distance between the sensor and the ground is constant. This shows that the air temperature cannot be assumed to be uniform.

Another simple correction method could be to use the average speed of sound in the air layer, assuming that the air temperature profile $\theta(z, t)$ varies linearly between the ground and the sensor, that is:

$$
\theta(z, t)=T_{g}(t)+\frac{T_{a}(t)-T_{g}(t)}{L} z
$$

where $L$ is the distance between the sensor and the ground, and $z$ is the altitude above the ground level. This method does not improve the accuracy of water level estimate beyond the naive correction methods introduced earlier. This analysis shows that more advanced methods are needed to estimate the distance between the sensor and the ground accurately.

These methods have to rely not only on current temperature measurements, but also on the history of measurements, resulting in a dynamical system. In this article, we investigate various models to compensate the thermal effects and correct distance measurements generated by the sensor. One of the simplest of such models involves a linear dynamical system with inputs, otherwise known as ARMAX (Auto Regressive Moving Average with Exogenous inputs) models, which we now investigate.

\section{B. Auto-regressive moving average exogenous (ARMAX) fit- ting}

ARMAX can be used for modeling time series and predicting future observations by combining a number of previous observations (Autoregressive), a number of noise terms (movingaverage), and a number of external (exogenous) inputs, which are $T_{a}(\cdot)$ and $T_{g}(\cdot)$ in the present case. Formally, the error $e(t)$ between the measured distance and the actual distance is given by:

$$
\begin{aligned}
& e(t)=\sum_{i=1}^{q} \varphi_{i} e(t-i)+\sum_{i=1}^{q} \theta_{i} \varepsilon(t-i)+ \\
& \sum_{i=1}^{q} \eta_{i} T_{a}(t-i)+\sum_{i=1}^{q} \xi_{i} T_{g}(t-i)+\varepsilon(t)
\end{aligned}
$$


where $\varepsilon(t)$ is the white noise, $\varphi_{i}, \theta_{i}, \eta_{i}$ and $\xi_{i}$ are the model parameters. $q$ is the number of terms considered in autoregressive, in moving-average and in exogenous inputs, or the order of ARMAX model. In our study, we set the order to a limited number of coefficients (20) in order to avoid overfitting, and to allow better comparison with other methods.

\section{Supervised learning}

Supervised learning has been extensively studied in machine learning and has been successfully applied to a wide range of applications. Given the inputs and outputs of a system, supervised learning algorithms model the functional relationship between inputs and outputs, with no need of knowing the system working principles. In other words, it can predict how the system reacts on the given inputs without knowing how a system works. In our study, the effects of the two temperature measurements provided by the sensors on the measured distance are too difficult to model accurately. Nevertheless, this complex relationship can be modeled through supervised learning, with inputs of the ambient and ground temperatures and outputs of the observed raw distance measurements from ultrasound sensors and the actual distance. Note that when floods do not occur (i.e. most of the time), the actual distance is perfectly known, and the output of the system is the raw distance measurement generated by the ultrasonic rangefinder. Once the relationship is learned, the actual distance between the sensor and the ground can always be estimated given the ambient and ground temperature measurements. Therefore, supervised learning can be viewed as a natural solution to our water level prediction problem.

1) Non-Linear regression: The first supervised learning method we used is non-linear regression, which models the target signal by a non-linear function. In this paper, the following quadratic function is utilized:

$$
e(t)=\sum_{i}\left(b_{3 i+1} T_{a}(t-k \cdot i)-b_{3 i+2} T_{g}(t-k \cdot i)+b_{3 i+3}\right)^{2}
$$

where $e(t)$ represents, as before, the difference between measured distance and actual distance at time $t . T_{a}(\cdot)$ and $T_{g}(\cdot)$ represent the ambient (sensor) and ground temperatures respectively, and $b$. are the parameters of the non-linear regression function. The combination $(k \cdot i)$ represents the discrete time horizon used for the prediction. To avoid over-fitting, we chose $k=5$, which results in a 15-parameter model taking into account the five most recent pieces of data. This number of parameters used in this model (15) is comparable to the 20 parameters used in the ARMAX analysis detailed above.

This quadratic regression function is selected after empirical comparison of models with different parameters. It is the best performing model that least suffers the overfitting problem and is more accurate on prediction. Also, the proposed model allows us to capture the influence of the two measured temperatures (ambient and ground temperatures) as well as the coupling (caused by the effects of solar irradiance, the heat island effect and the presence of shadows on the ground) between them. The fitting is done through successive approximations, for which we limit the number of iterations to 100 and the tolerance to $10^{-8}$. Robust fitting options can be added to improve the root mean square error performance of the predicted values.

2) Neural networks: Artificial Neural Networks (ANN) are a computational model inspired by the central nervous systems of animals [29]. This concept and a few possible applications in industrial electronics are summarized in [30], [31]. In this specific field, there already exist a large number of applications of neural networks, some of which include motor drives [32] or power distribution problems dealing with harmonic distortion. Due to their nonlinear nature, they have also become an integral part of the field of control systems engineering [33], [34].

a) Neural network architecture: Neural networks can be considered as a combination of a set of nonlinear functions that are organized structurally layer by layer. They are thus also called MLPs (Multilayer perceptron). They are well suited to various application problems due to their "universal approximation" property: any continuous function can be uniformly approximated to an arbitrary accuracy by neural networks, given enough hidden units with any of a wide variety of continuous nonlinear hidden-layer activation functions, see for instance [35], [36], and [37].

In our problem setting, there are two inputs (ambient and ground temperatures), and one output (the raw distance measurement from the ultrasound). Each neuron in the hidden layer connecting to the inputs is fed with the input variables $x_{1}, \ldots, x_{D}$. The complete network function is obtained by:

$$
y_{k}(x, \mathrm{w})=\alpha\left(\sum_{j=1}^{M} w_{k j}^{(2)} h\left(\sum_{i=1}^{D} w_{j i}^{(1)} x_{i}+b_{j 0}^{(1)}\right)+b_{k 0}^{(2)}\right)
$$

where $j=1, \ldots, M$ ( $M$ is the total number of hidden neurons in the this layer). The parameters $w_{j i}^{(1)}$ and $b_{j 0}^{(1)}\left(w_{k j}^{(2)}\right.$ and $\left.b_{k 0}^{(2)}\right)$ are the weights and the biases for the inputs to the first (second) hidden layer. $h($.$) is an activation function (in the present case$ the activation functions are sigmoid functions). The output is a function $\alpha($.$) (which will be discussed in section III-C2b) of$ the second layer variables. Thus the neural network model can be considered as a nonlinear function mapping a set of input variables $\left\{x_{i}\right\}$ to a set of output variables $\left\{y_{k}\right\}$ controlled by a vector w of adjustable parameters.

b) Levenberg-Marquardt back-propagation training function: Training a neural network involves the tuning of the weights and biases of the network. The objective is to maximize the network prediction performance, which corresponds to minimizing the difference between all network outputs $y_{k}$ and desired outputs or targets $t_{k}$ on validation data. The computational time required for the training algorithm depends on many factors, including the complexity and objective function of the problem, the size of training data, the number of parameters in the network, and whether the network is being used for pattern recognition (discriminant analysis) or function approximation (regression [36]). For our particular problem, we are interested in the function approximation problem with a few hundred weights in a moderate size network. In this specific case, the Levenberg-Marquardt algorithm has been proven to have the fastest convergence [38]. It updates the 
network weights and biases in the direction in which the performance function decreases most rapidly. This advantage is more noticeable when implementing $\mathrm{NN}$ on the proposed platform of this work, since sensors have a limited bandwidth, and that transmitting all the data would be energy intensive, thus it is very critical to consider efficient online training.

Having stated all of this and tried different modules. We realized that we need an efficient preprocessing tool in addition to the online training. Indeed, the raw data generated by the infrared temperature sensors and the ultrasound rangefinder have different scales, and sometimes exhibiting inconsistencies, as can be observed in Figure 3 which is a major game changer in our application. Therefore, an effective preprocessing procedure is essential in the accurate sensing of floods based on measurement data produced from sensor nodes.

\section{PROposed SOLUTION AND SySTEM PERFORMANCE}

\section{A. Preprocessing of measurement data}

As stated previously in section III, the raw data generated by the infrared temperature sensors and the ultrasound rangefinder have different scales, and are sometimes exhibiting inconsistencies. We propose a preprocessing procedure as illustrated in Figure 5. It mainly involves two main processes: fault detection and missing data reconstruction.

1) Sensor fault detection: Sensor faults can be caused by multiple factors. In the present case, these faults were mainly due to gateway failures (due to a loss of the internet connection), and brownouts of the sensor caused by faulty charging circuits in a narrow solar power input range when collecting the measurement data.

We first check the network faults (communication faults), which are identified by detecting time periods that have more than 15 minutes (i.e. 90 samples at the $0.1 \mathrm{~Hz}$ message update rate) without reception of data from sensors. When such faults are detected, the missing data in the blank periods are reconstructed directly by the LASSO-based formulation (least absolute shrinkage and selection operator) which will be explain in the following section. To remove outliers (caused by vehicles passing below the sensor), we apply a moving median filter to the temperature and distance data. Finally, we detect sensor fault by analyzing the consensus among all six infrared temperature sensors in the node. Whereas for the ambient temperature and the distance measurements the consensus is not applicable since we have one of each in a sensor node. We then use a sliding window, in which we calculate the mean $\mu$ and the standard deviation $\sigma$ of the measurement data of all the sensors. The records that deviate more than $3 \sigma$ from the mean are considered anomalies, and excluded from the dataset. Since the main cause of sensor noise is thermal noise [39], the sensor measurements follow a normal distribution, given that the thermal anomalies caused by the vehicles are suppressed by the median filter. Hence, $99.7 \%$ of the values fall within the $[\mu-3 \sigma, \mu+3 \sigma]$ window. Most of the data excluded using this approach is indeed faulty data. All data gone through the fault detection process are stored and served to the next step, approximating the missing data to complete the measurements.
2) $L_{1}$ regularized reconstruction: In order to reconstruct the missing data in real-time, we leverage the data generated during the previous days as follows. Let us consider the time series $s_{i}(\cdot)$, obtained during the previous $i \in\left\{0, \ldots, i_{\max }\right\}$ days, and let $j \in\left\{0, \ldots, j_{\max }\right\}$ represent a time index in a day (in practice, $j$ ranges from 0 to 8640 since we have a sampling rate of $0.1 \mathrm{~Hz}$ ). We assume that the current measurements $s(j)$ can be written as a linear combination of the past measurements, as follows:

$$
s_{\text {estimate }}(j)=\sum_{i=0}^{i_{\max }} \alpha_{i} s_{i}(j)
$$

where $\alpha_{1}, \cdots, \alpha_{i_{\max }}$ are model parameters. Finding these parameters can be done by minimizing the estimation error, for example in the least squares sense:

$$
\min _{\alpha} \sum_{j \in J}\left(\sum_{i=1}^{i_{\max }} \alpha_{i} s_{i}(j)-s_{0}(j)\right)^{2}
$$

where $J \subset\left\{0, \ldots, j_{\max }\right\}$ is the set of times (for the current day) associated with non-faulty measurements. $s_{0}$ is the current day measurements.

In general, $|J|$ is considerably larger than $i_{\max }$, and the problem is overdetermined. The main issue with this formulation is the fact that any given day should only have a few identifiable features from the previous days patterns, and thus, $\alpha$ should be sparse. To enforce this sparsity constraints, we add a $L_{1}$ norm regularization term to the above formulation, leading to the formulation of our estimation problem as a LASSO [40]:

$$
\min _{\alpha} \sum_{j \in J}\left(\sum_{i=1}^{i_{\max }} \alpha_{i} s_{i}(j)-s_{0}(j)\right)^{2}+\lambda\|\alpha\|_{1}
$$

where $\lambda$ is the regularization parameter that trades off fitting quality and sparsity. Sparsity also improves the robustness of the regression to noise.

Problem (7) is a quadratic program (QP) which can be solved using standard convex optimization methods. The results of the preprocessing scheme is illustrated in Figure 6. In this Figure, we show the raw measurement data, along with the output of the fault detection stage, and the output of the LASSO-based data reconstruction stage. We applied the above algorithms to filter measurement data over extended periods of time. Figure 7 illustrates the reconstructed measurement data between December 4th 2013 and December 21st 2013, which is used as training data for the artificial neural network-based water level estimation scheme, introduced in the subsequent sections.

This section reports the performance of different models we studied in the previous section. The data set used in this validation has been generated from the sensors deployed on our university's campus.

\section{B. Artificial neural networks performance}

The number of neurons has been set to achieve good performance on the validation dataset, considering the trade off between the computational time required to train the network 


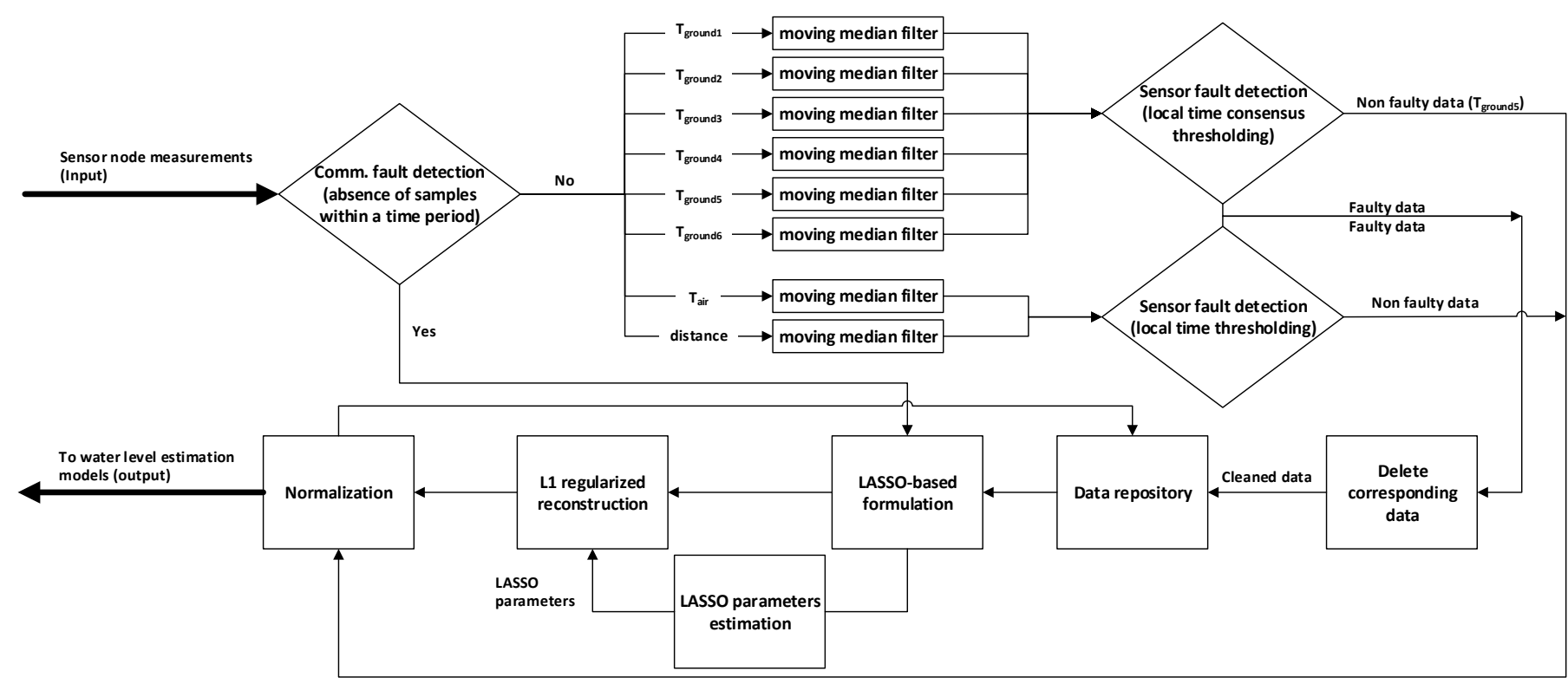

Figure 5: The preprocessing of the measured data from the sensor node. The processed data are served to the estimation models.

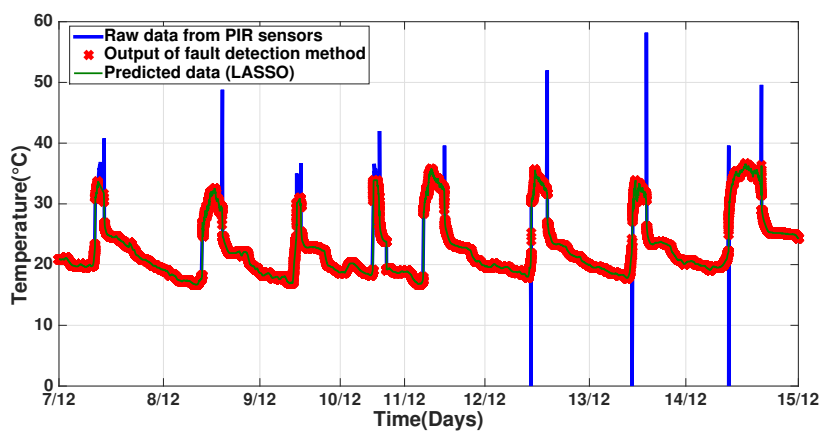

Figure 6: Comparison between raw PIR sensor data (blue) and reconstructed PIR data (green) at the end of the preprocessing stage for a sample of the data in December (without normalization).

and the performance of root mean square error (RMSE) that can be reached. Furthermore, we had to fix the number of neurons to be comparable with the other methods we used to estimate the water level (since the number of neurons determines the number of coefficients to be optimized). RMSE only improves marginally as we increase the number of neurons past 5 neurons, as can be seen from Figure 8.

We implemented the neural network model using the neural network toolbox of Matlab, running on a Mackbook i7. We were able to evaluate and train the neural network using the Levenberg-Marquardt algorithm with one hidden layer of 10 neurons in a two inputs and one output setting.

The dataset consists of 205000 data points, as illustrated in

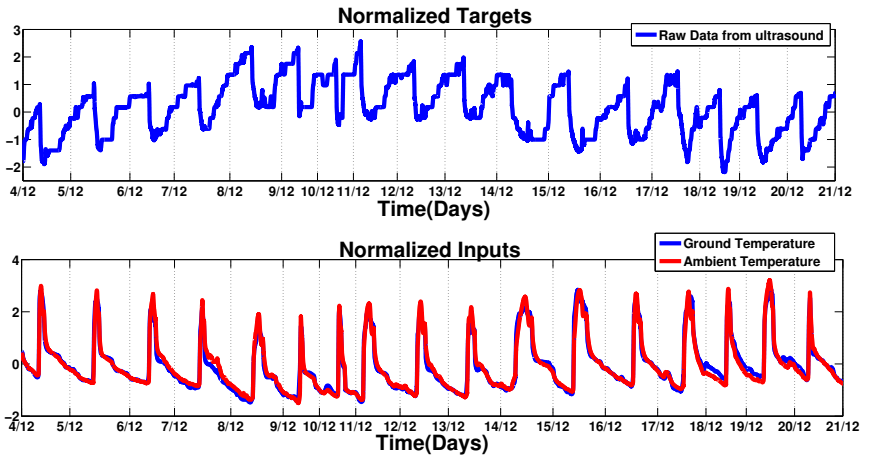

Figure 7: Top: Normalized and filtered raw distance measurements (for the preprocessed training dataset). Bottom: Normalized and filtered ambient and ground temperature measurements (for the preprocessed training dataset).

Figure 7. We arbitrarily divided it into a training dataset, a validation dataset and a testing dataset. The training dataset corresponds to $70 \%$ of the overall data, while the validation and testing datasets correspond to $15 \%$ of the data each. The validation dataset is used during the training process, to halt training when the performance of the estimation on the validation dataset stops improving.

Figure 9 shows the histogram of the error between output and targets for the training, validation and testing samples. It can be seen in the figure that the histogram fits a normal distribution with a maximum detected error on validation and on testing samples of less than $2 \mathrm{~cm}$. 


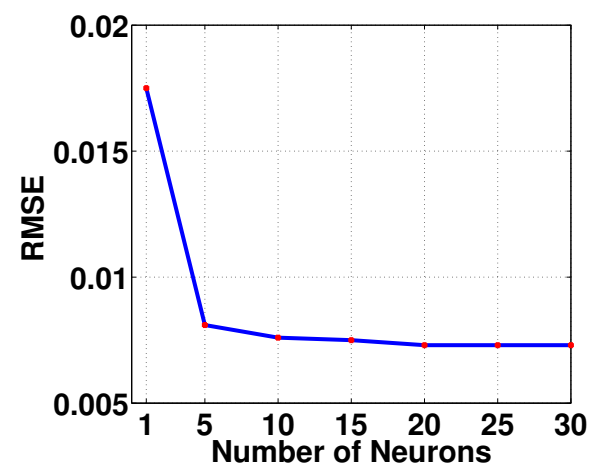

Figure 8: Relation between the number of neurons in the hidden layer of an $\mathrm{ANN}$ and the root mean square value (RMSE). As can be seen from this Figure, the RMSE only improves marginally as we increase the number of neurons past 5 neurons.

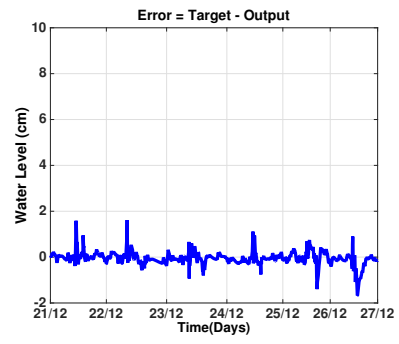

(a)

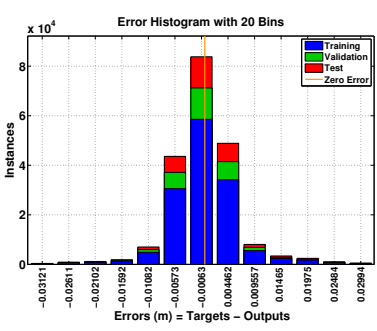

(b)
Figure 9: (a) Water level prediction during a week of December 2013 for the sensor node "A77D". Since no flood occurred during this period, the actual water level was always $0 \mathrm{~cm}$. The deviation of predicted value from 0 can be considered as prediction error, which is less than $1.85 \mathrm{~cm}$. (b) Error distribution over the training, testing and validation datasets.

The absolute difference (so-called Mean Absolute Error, $\mathrm{MAE}$ ) between predicted and actual distance measurements is shown in Figure 9. This difference is also considered as the prediction of water level. Since during this period (December 21 st to 27th) no flood occurred, the actual water level was always $0 \mathrm{~cm}$. As can be seen from the figure, the deviation of prediction from 0 is less than $1.85 \mathrm{~cm}$. This low prediction error shows the good performance of a neural network on water level prediction.

\section{Comparison of prediction models}

Table I shows the comparison between all the approaches investigated in this article for water level estimation, on an identical dataset (i.e., similar range of validation dataset from December 21st to 27th, 2013, and when training is required we used similar inputs, preprocessed data, shown in Figure
7). Their performance is compared in terms of prediction accuracy (measured by the maximum absolute error $\left(L_{\infty}\right)$ and RMSE), efficiency (computational time in both training and prediction) and prediction stability (standard deviation of errors in 5 independent runnings of the same testing dataset).

Besides naive compensation and ARMAX, our proposed neural network approach is compared to three other machine learning techniques, non-linear regression, decision tree and fuzzy logic, which are widely used supervised learning methods for making predictions [41]. The neural network and nonlinear regression are introduced in section III-C1 and III-C2, respectively. We designed a fuzzy logic predictor using two membership functions on our two inputs, and thus resulting in four fuzzy rules. Then we use a hybrid learning algorithm to tune the parameters of a Sugeno-type fuzzy interference system [42] which is a combination of the least-squares and back-propagation gradient descent methods to model a training data set. For comparison purposes, we ensured that the number of parameters to optimize is similar to the other models and the same training and testing data are used for evaluation. Decision tree for regression is employed for estimating water level at leaf nodes, while each branching node splits values of one input variable. The tree is trained by the "Regression Tree" functions provided in Matlab, with the same training data we used for the other models.

On accuracy, the four machine learning (ML) methods presented in this work have performed better than the naive compensation and ARMAX, in which the neural network (NN) approach performs the best. With regard to computational time, the naive compensation is associated with a zero training time, while the training time for the ARMAX estimation method is negligible (since the model is linear). Each of the ML methods involves a training process, and thus demands some time for learning. However, it is more important to compare the computational time in prediction, because ML training can always be conducted offline. Except for ARMAX, the five approaches need around 5-6 seconds to make the prediction for the complete testing dataset (i.e. $30 \%$ of the complete dataset of 205000 data points), that is, $8.76 \times 10^{-5}$ seconds to make one prediction, which is fast enough to satisfy the real time requirements.

The ML methods are also more stable than others, and thus can guarantee that the prediction is reliable.

Table I: Comparison of the performance of 6 models

\begin{tabular}{|l|l|l|l|l|l|}
\hline Model & \multirow{2}{*}{$L_{\infty}(\mathrm{cm})$} & \multirow{2}{*}{ RMSE } & \multicolumn{2}{|l|}{ Computing time (sec.) } & \multirow{2}{*}{ Error Std. } \\
\cline { 4 - 5 } & & & Training & Prediction & \\
\hline Naive Compensation & 15.3 & 0.0547 & 0 & 5.3 & 0.0504 \\
\hline ARMAX & 4.9 & 0.0164 & 0 & 272.3 & 0.0152 \\
\hline Fuzzy Logic (ML) & 2.59 & 0.0413 & 168.6 & 5.5 & 0.0377 \\
\hline NL Regression (ML) & 2.57 & 0.0082 & 28 & 5.7 & 0.0083 \\
\hline Decision Trees (ML) & 2.15 & 0.0158 & 26.6 & 5.9 & 0.0158 \\
\hline NN (ML) & 0.6 & 0.0058 & 975 & 5.2 & 0.006 \\
\hline
\end{tabular}

\section{Minimization of errors in the $L_{\infty}$ sense}

Most ML techniques involve the minimization of the prediction error in the $L_{2}$ norm sense, as we investigated above. Since 
flash floods are extremely rare events, we are also interested in making prediction with minimized error in the $L_{\infty}$ sense, to avoid false alarms in the flood monitoring system. The infinity norm is better than norm 2 for the task, since we want to detect events based on thresholding, and a very low norm 2 (but high norm infinity) in the training (non flood) data is unacceptable, since it would lead to false detections.

Our objective in this subsection is thus, technically to minimize the prediction error in the $L_{\infty}$ sense instead of the $L_{2}$ norm sense, though the $L_{\infty}$ error usually improves when the $L_{2}$ error is reduced.

Minimizing the prediction error in the $L_{\infty}$ sense is straightforward in the case of a linear model: it can be formally written as:

$$
\min _{W}\|X W-Y\|_{\infty}
$$

where $X$ is the input, $W$ is the model coefficient to be optimized, and $Y$ is the output. This resulting Linear Program (LP) problem is solved by the SDPT3 package working under Matlab in this paper.

We investigate both the linear model (ARMAX) and the nonlinear model (non-linear regression) for minimizing error in $L_{\infty}$. Table II shows the performance of ARMAX and nonlinear regression, measured in maximum absolute difference $\left(L_{\infty}\right)$, RMSE and standard deviation of errors (in 5 independent runnings of the same testing dataset). Comparing the $L_{\infty}$ values of these two methods in Table II and Table I, we can see that they have smaller $L_{\infty}$ when minimizing $L_{\infty}$ error than when minimizing $L_{2}$ error. The non-linear regression model with minimized $L_{\infty}$ error is comparable to the ANN model with minimized $L_{2}$ error, which is the best as observed in Table I.

With regard to the RMSE, both ARMAX and non-linear regression have high error value when minimizing error in $L_{\infty}$. This is understandable, as, by nature, they lead to small RMSE (a performance metric defined in $L_{2}$ ) when minimizing error in $L_{2}$.

Table II: Comparison between ARMAX and NL regression when minimizing error in $L_{\infty}$

\begin{tabular}{|l|l|l|l|}
\hline Model & $L_{\infty}(\mathrm{cm})$ & RMSE & Error Std. \\
\hline ARMAX & 2.28 & 1.2593 & 1.0128 \\
\hline NL Regression (ML) & 0.55 & 0.3218 & 0.2572 \\
\hline
\end{tabular}

\section{E. Temporal robustness of ANN model parameters}

In this section, we evaluate the time dependence of the training parameters. For this, we train the artificial neural network model using data from the early December (December 4th to 21st), and validate the performance on three different sets of data (temporally separated), namely late December (December 21st to 27th), January and February.

Table III shows the performance of the estimation models of the neural networks on the sensor node "A77D" using training data from early December 2013 only. As can be noted, the prediction performance slightly degrades in February. However, the estimation error is acceptable (within about $2 \mathrm{~cm}$ ) for flash flood monitoring applications. This suggests that the coefficient can be used for large durations (e.g., tens of days) before degrading (hence it needs to be tuned), which enables large datasets to be integrated in the training process.

Table III: Evaluation of temporal robustness: comparing prediction accuracy in different months when using NN trained by data in early December, 2013.

\begin{tabular}{|l|l|l|l|}
\hline Month & $L_{\infty}(\mathrm{cm})$ & RMSE & Error Std. \\
\hline Late December & 1.63 & 0.0215 & 0.0213 \\
\hline January & 2.08 & 0.0267 & 0.0267 \\
\hline February & 2.15 & 0.0272 & 0.0272 \\
\hline
\end{tabular}

\section{F. Spatial robustness analysis of ANN model}

To evaluate the robustness of the model parameters with respect to spatial changes, we installed two sensor nodes in a close proximity (during January 2014): sensors "A77D" and "8F90". Node "8F90" is placed in an area that can be shadowed by a building at some times of the day, unlike node "A77D".

In a similar manner to the analysis done to "A77D", we estimated the water level and the norm infinity error of estimation during a period of 11 days is shown in Figure 10 (picked right after we installed the node early in January 2014). It can be seen from Figure 10 that the error is less than 0.6 $\mathrm{cm}$.

Then, in order to evaluate the spatial robustness, the neural network is trained by data from sensor "A77D" during December 4th and 21st, 2013, to predict the data for the sensor node "8F90" over the same testing period of the 11 days.

As expected, the performance of the prediction of "8F90" slightly degrades in this case: RMS error of 0.275 vs. 0.0058 and norm infinity error of $1.735 \mathrm{~cm}$ vs. $0.6 \mathrm{~cm}$ for the training with the data of "8F90", compared to the training with the data of "A77D". This acceptable error (i.e. less than $2 \mathrm{~cm}$ ) allows us in practice to use training data from the nearest sensors to immediately generate distance measurement data from a newly installed sensor, without having to wait for a training period.

\section{G. Validation on an actual flooding incident}

Flood incidents are usually rare, but one such incidents occurred in the test period, the only incident which has occurred to date. This incident has been detected by two sensors installed in Umm Al Qura University in Mecca, Saudi Arabia, where climate conditions differ and floods are more frequent. The flash flood occurred on the 8th of May 2014, and, fortunately, did not result in extreme damage and caused only a single casualty [43]. Students at the university reported that the street water level was locally around $10 \mathrm{~cm}$, and the incident started at around 11PM local time.

This incident has been captured by the two sensors "8F48" and "D3CB". The water level estimates (using the days before May 6th as training data for both sensors) are illustrated in Figure 11 for both sensors. As can be seen from this Figure, the flood is clearly detected, and corresponds to a significant 


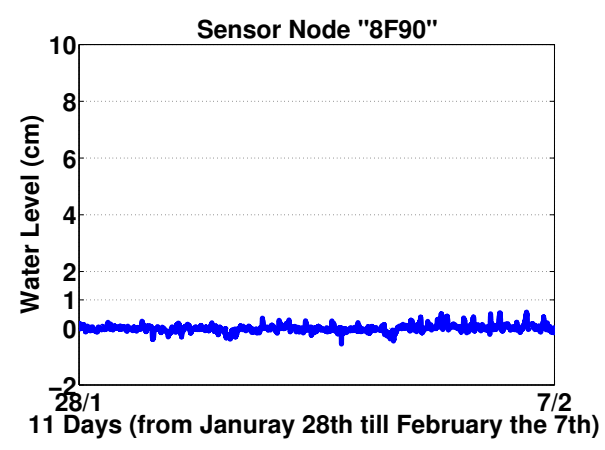

Figure 10: Estimated water level at the sensor node "8F90" from January 28 to February 7, 2014. In this test, the artificial neural network is trained onboard by the sensor node itself.

rise in the water level estimate. We can also see the onset of the flood is identical for both sensors, and that the estimated water levels are of the same order of magnitude (7 to $9 \mathrm{~cm}$ ).

Unfortunately, the sink node has failed shortly after the flood occurred, around 11:30 PM. The failure of the sink node was probably caused by an electrical failure related to the flooding event. Nevertheless, the available data clearly shows the detection of a minor event shortly before midnight, 9th of May 2014, on both sensors. There was no way to detect the
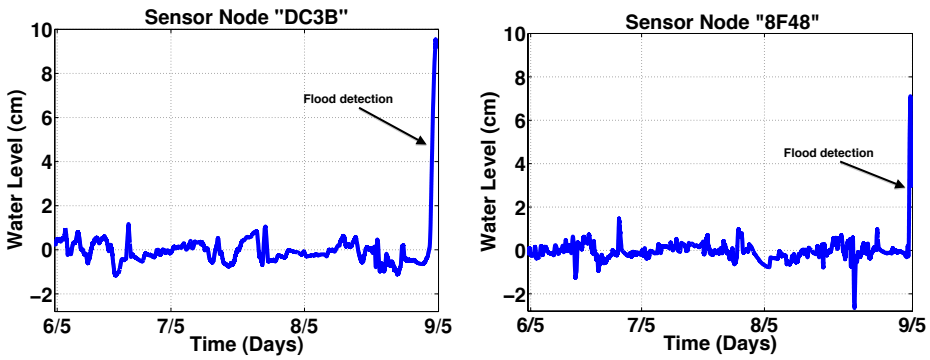

Figure 11: Water level estimation between May 6 and May 9, 2014, for sensor nodes "DC3B" and "8F48" deployed in Umm Al Qura University campus.

flood incident from the raw data of the ultrasonic, as illustrated in Figure 12 for one of the sensor nodes that encountered the flood (i.e. "8F48"). As shown by this Figure, naive thresholding on the raw water level measurement data would not allow one to detect the flood.

\section{IMPLEMENTATION OF ANN ALGORITHMS ON MICROCONTROLLERS}

In order to validate our design and be able in practice to use our solution, we implement our algorithms on the dedicated microcontroller board and validate the performance as explained in the following sections.
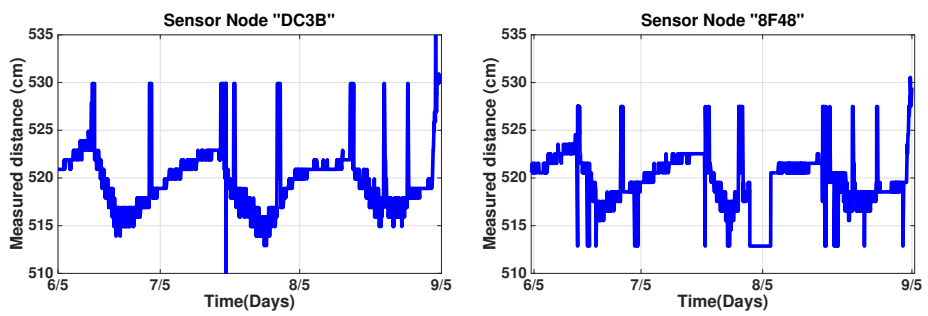

Figure 12: Raw ultrasonic measurements between May 6 and May 9, 2014, for sensor nodes "DC3B" and "8F48" deployed in Umm Al Qura University campus.

\section{A. Dedicated sensing platform for flash flood monitoring ap- plications}

In the present case, we have built a customized hardware platform, described in the companion article [26]. This platform is built around a 32-bit microcontroller and illustrated in Figure 13. We selected for this application, the STM32F407, a 32-bit ARM Cortex-M4 based microcontroller from ST Microelectronics since it satisfies the requirements of our estimation problem and best trades off RAM, power consumption, cost and most importantly for this study, computation (since this sensor is sensing traffic flow, relaying and forwarding other messages in the sensor network, and estimating traffic flow conditions [25]).

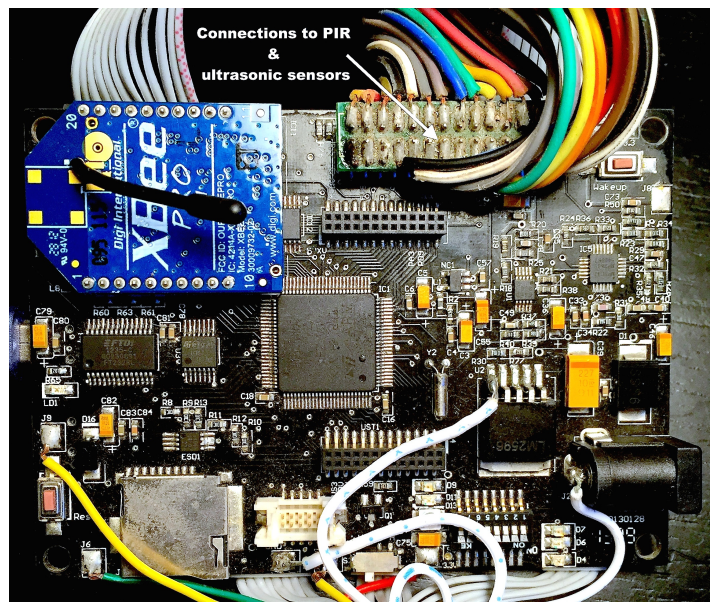

Figure 13: Custom-developed 32-bit microcontroller platform $(9 \mathrm{~cm} \times 6.5 \mathrm{~cm})$ connected to an XBee module and to PIR and ultrasonic sensors.

\section{B. On-Board Neural Network Algorithm}

In order to build a neural network on the custom-based microcontrollers, we need to build not only the architecture but also the data storage system, for performing both training and prediction. The key values to be stored are weights associated 
with neurons, the weight updates and error gradients during the training phase.

1) Initialization of the Neural Network: To minimize the number of iterations in neural network training before convergence, the weights associated with neurons should be carefully initialized. Since our simulation results from training data set already show good estimation performance, and good spatiotemporal robustness, as shown in sections IV-E and IV-F, the weights of the trained neural network of any given sensor is a good initial guess. Therefore, we initialize the neural network on board by the weights of the neural network trained in the simulation from training data between December 4th till December 21st.

2) Neural Network Training: As we have illustrated previously in section IV-E, and in Figure 14, the trained NN model is robust when it is applied to long term prediction over two months until the error propagates due to the weather change. In order to continuously make accurate prediction, the NN model should be updated with the recently received data, which are saved in a 2 GB micro SD card. The card has the capacity to host at most 12 months of data, which are sufficient for NN updating. Due to the limited computing power on

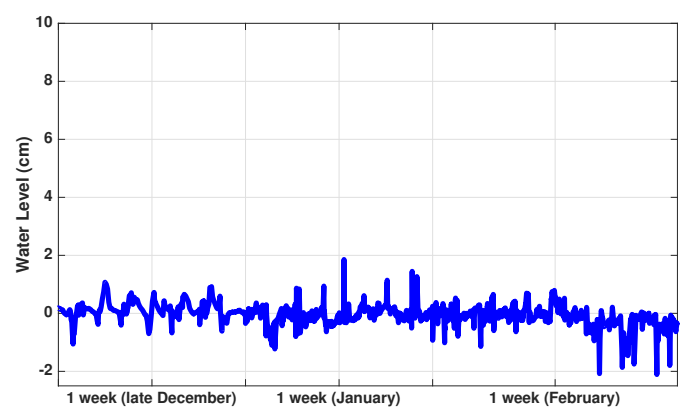

Figure 14: Prediction accuracy during different months on the sensor node "A77D" installed on our campus in November 2013, using training data from December 2013 only. As can be seen from this Figure, the prediction performance slightly degrades in February, though its performance is still acceptable for flash flood monitoring applications.

board, updating the NN cannot be implemented in the batch mode introduced in section III-C2b. The main reason is the repeatedly heavy computation of $y_{k}$ in Equation 4 for all $x$ in training data during the error propagation process. Thus we adopt the online training mode for updating the weights in NN model, due to its comparable performance to the batch mode but better efficiency. Instead of taking all $x$ in training data for a single update of weights in $\mathrm{NN}$, the online mode updates the weights by using $x$ one by one. That is to say, the data saved in SD card flow into the NN individually and update it until the prediction error is reduced down below an acceptable threshold (e.g., $2 \mathrm{~cm}$ in the $L_{\infty}$ case).

3) Implementation: The implementation of code is done using Keil $\mathrm{V} 4.7$ [44] which is a software provided by the $\mathrm{ARM}$ group, and optimized for $\mathrm{C} / \mathrm{C}++$ language. We have implemented our algorithms on the wireless sensor nodes using a conventional back-propagation neural network class in C language that makes use of gradient descent, with parameters defined as: 0.001 learning late, 1500 of maximum epochs during training, and maximum accuracy. The demonstration code is a 996 lines written in $\mathrm{C}++$ language and is built on top of $\langle$ math.h>, <algorithm>, <fstream>, <strings, <vector>, <stdio.h> libraries, as well as the designed neural network class "neuralnetwork. h". The code can toggle between batch and online training mode, and gets the training stopping criteria from the user. Its total memory size (when compiled) is $101 \mathrm{kB}$ (our microcontroller's ROM size is $1 \mathrm{MB}$ ), while its peak memory usage is $58 \mathrm{kB}$ (our microcontroller total RAM size is $192 \mathrm{kB}$ ), well below the limits of the Cortex M4 microcontroller.

4) Neural Network performance: We coded the neural network algorithm on the custom-based microcontroller platform described earlier, and tested them for real time performance. The prediction performance of the NN running on the computational platforms has similar results to the ones obtained on Mat lab. However, since the platform has less computational capabilities than a computer, its computational time was expected to be much greater when we consider the training in addition to the prediction of the data in order to converge to the solution. We show the performance on the prediction of water level with retraining when the threshold is exceeded at the beginning of February 2014 in Figure 15. We used one week of data following the online training approach to retrain the NN parameters and as we can see this helped bringing the error to within $2 \mathrm{~cm}$. The computational time measured for the process of training and predicting a week of data onboard with the online training approach took approximately, 118 minutes (11.2 minutes for prediction). We implement the onboard NN on the custom-based microcontroller platform, and evaluate its performance to see whether prediction error can be reduced after online updating. In Figure 14, we show the performance on the prediction of water level with training data from December 2013 only. It can be seen that the prediction performance slightly degrades in February 2014 (sometimes with errors greater than $2 \mathrm{~cm}$ ). In Figure 15, we show the prediction with online training when the error exceeded $2 \mathrm{~cm}$ at the beginning of February 2014. We used one week of data following the online training mode to update the NN parameters. We can see that the error is reduced to be less than $2 \mathrm{~cm}$.

Due to the limited computational capability of the lowpower platform, the online training mode took about 2 hours for absorbing the one week data, while the prediction is quite fast 0.03 seconds per data sample. Therefore, the online training employed only one week data and stopped to proceed with more data to further reduce the error that is already less than $2 \mathrm{~cm}$.

\section{DISCUSSION}

The recent vast research activities in neural classification have established that neural networks are a promising alternative to various conventional classification methods. The 


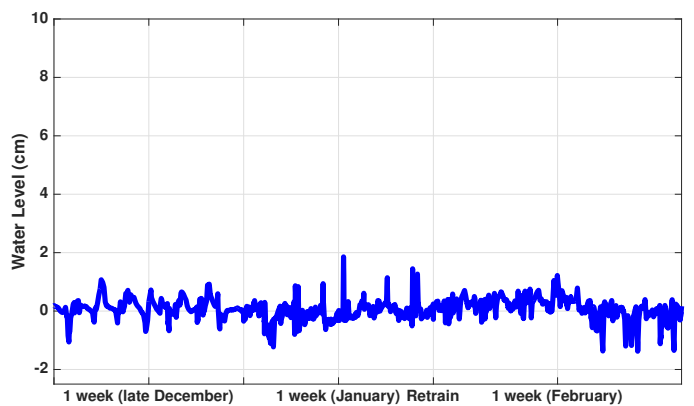

Figure 15: Prediction accuracy during different months on the sensor node "A77D" installed on our campus in November 2013, using training data from December 2013, with online on-board retraining of the parameters early February 2014. As can be seen from this Figure, the prediction performance has improved in February, compared to the results shown in Figure 14.

advantage of neural networks lies in the following theoretical aspects. First, neural networks are data driven self-adaptive methods in that they can adjust themselves to the data without any explicit specification of functional or distributional form for the underlying model. Second, they are universal functional approximators in that neural networks can approximate any function with arbitrary accuracy. Third, neural networks are nonlinear models, which makes them flexible in modeling real world complex relationships. Finally, neural networks are able to estimate the posterior probabilities, which provides the basis for establishing classification rule and performing statistical analysis.

In the present case, ANNs exhibit good convergence properties, which enables us to use a low number of neurons and layers, making it suitable to a low power embedded systems application. In future work, we will investigate the possibility of using ANNs for detecting other information related to flooding, for example, the presence of water on the ground, or the presence of rain, which may be inferred from air and ground temperature measurement patterns. Preliminary analysis on the flood data that we collected shows the possibility of using ANNs in this context, though more validation is needed.

\section{CONCLUSION}

In this article, an ANN approach is presented to contribute to the literature of flash flood sensing using a custom-designed sensor comprising an ultrasonic rangefinder and multiple passive infrared temperature sensors. Because of the extremely low absolute distance measurement error required by the system (on the order of $0.2 \%$ ), one needs to estimate the temperature profile of the air layer between the sensor and the ground, as this temperature affects the speed of sound. Since this profile is impossible to model accurately (because of unknown model parameters and unknown boundary conditions), a non modelbased approach is chosen for estimating the correction due to deviations in temperature showing that ANNs capture the effects of the underlying model very accurately, better than nonlinear regressions, fuzzy-based models or decision trees. ANNs can be used to monitor water level in streets in real time, given the important preprocessing step that contributes substantially to the accuracy of the estimation. This approach has been validated during an extensive testing period, and demonstrated high precision and robustness. Such algorithms can run in real time on low-power microcontroller platforms, greatly reducing power usage and bandwidth requirements in a wireless sensor network context as demonstrated in this work. Future work will be focused on the detection of rain using the reflections of the secondary lobes of the ultrasonic rangefinder. We will also investigate the detection of water presence (not depth) on the ground using air and ground temperature measurements. This water presence information can be used for fault detection purposes, to make sure that the change in ground distance is actually caused by water.

\section{REFERENCES}

[1] Mustafa Mousa and Christian Claudel. Energy parameter estimation in solar powered wireless sensor networks. In Real-World Wireless Sensor Networks, pages 217-229. Springer, 2013.

[2] Nagender Kumar Suryadevara and Subhas Chandra Mukhopadhyay. Wireless sensor network based home monitoring system for wellness determination of elderly. Sensors Journal, IEEE, 12(6):1965-1972, 2012.

[3] Matteo Ceriotti, Luca Mottola, Gian Pietro Picco, Amy L Murphy, Stefan Guna, Michele Corra, Matteo Pozzi, Daniele Zonta, and Paolo Zanon. Monitoring heritage buildings with wireless sensor networks: The torre aquila deployment. In Proceedings of the 2009 International Conference on Information Processing in Sensor Networks, pages 277288. IEEE Computer Society, 2009.

[4] Adrian Burns, Barry R Greene, Michael J McGrath, Terrance J O'Shea, Benjamin Kuris, Steven M Ayer, Florin Stroiescu, and Victor Cionca. Shimmer-a wireless sensor platform for noninvasive biomedical research. Sensors Journal, IEEE, 10(9):1527-1534, 2010.

[5] Ahmad H Dehwah, Mustafa Mousa, and Christian G Claudel. Lessons learned on solar powered wireless sensor network deployments in urban, desert environments. Ad Hoc Networks, 28:52-67, 2015.

[6] Koen Langendoen, Aline Baggio, and Otto Visser. Murphy loves potatoes: Experiences from a pilot sensor network deployment in precision agriculture. In I Proceedings 20th IEEE International Parallel \& Distributed Processing Symposium, page 155. IEEE, 2006.

[7] Cesare Alippi, Romolo Camplani, Cristian Galperti, and Manuel Roveri. A robust, adaptive, solar-powered wsn framework for aquatic environmental monitoring. Sensors Journal, IEEE, 11(1):45-55, 2011.

[8] Hsueh-Chun Lin, Yiao-Chiang Kan, and Yao-Ming Hong. The comprehensive gateway model for diverse environmental monitoring upon wireless sensor network. Sensors Journal, IEEE, 11(5):1293-1303, 2011.

[9] Liqun Hou and Neil W Bergmann. Novel industrial wireless sensor networks for machine condition monitoring and fault diagnosis. Instrumentation and Measurement, IEEE Transactions on, 61(10):2787-2798, 2012.

[10] Michael K Lindell and Carla S Prater. Assessing community impacts of natural disasters. Natural Hazards Review, 4(4):176-185, 2003.

[11] ISDR disaster statistics, http://www.unisdr.org/.

[12] Sebastiaan N Jonkman and Ilan Kelman. An analysis of the causes and circumstances of flood disaster deaths. Disasters, 29(1):75-97, 2005.

[13] PCD Milly, RTi Wetherald, KA Dunne, and TL Delworth. Increasing risk of great floods in a changing climate. Nature, 415(6871):514-517, 2002 . 
[14] M Castillo-Effer, Daniel H Quintela, W Moreno, R Jordan, and W Westhoff. Wireless sensor networks for flash-flood alerting. In Devices, Circuits and Systems, 2004. Proceedings of the Fifth IEEE International Caracas Conference on, volume 1, pages 142-146. IEEE, 2004.

[15] Endrowednes Kuantama, Leonardy Setyawan, and Jessie Darma. Early flood alerts using short message service (sms). In System Engineering and Technology (ICSET), 2012 International Conference on, pages 1-5. IEEE, 2012.

[16] CL Lai, JC Yang, and YH Chen. A real time video processing based surveillance system for early fire and flood detection. In Instrumentation and Measurement Technology Conference Proceedings, 2007. IMTC 2007. IEEE, pages 1-6. IEEE, 2007.

[17] Hongyan Fu, Xuewen Shu, Aping Zhang, Weisheng Liu, Lin Zhang, Sailing $\mathrm{He}$, and Ian Bennion. Implementation and characterization of liquid-level sensor based on a long-period fiber grating mach-zehnder interferometer. Sensors Journal, IEEE, 11(11):2878-2882, 2011.

[18] Ni-Bin Chang and Da-Hai Guo. Urban flash flood monitoring, mapping and forecasting via a tailored sensor network system. In Networking, Sensing and Control, 2006. ICNSC'06. Proceedings of the 2006 IEEE International Conference on, pages 757-761. IEEE, 2006.

[19] Mustafa Mousa, Enas Oudat, and Christian Claudel. A novel dual traffic/flash flood monitoring system using passive infrared/ultrasonic sensors. In Mobile Ad Hoc and Sensor Systems (MASS), 2015 IEEE 12th International Conference on, pages 388-397. IEEE, 2015.

[20] Mustafa Mousa and Christian Claudel. Water level estimation in urban ultrasonic/passive infrared flash flood sensor networks using supervised learning. In Proceedings of the 13th international symposium on Information processing in sensor networks (IPSN), pages 277-278. IEEE Press, 2014.

[21] VE Sakharov, SA Kuznetsov, BD Zaitsev, IE Kuznetsova, and SG Joshi. Liquid Level Sensor Using Ultrasonic Lamb Waves. Ultrasonics, 41(4):319-322, 2003.

[22] Richard Hunter Brown. Liquid Level Sensor, December 16 1997. US Patent 5,697,248.

[23] Elizabeth A Basha, Sai Ravela, and Daniela Rus. Model-Based Monitoring for Early Warning Flood Detection. In Proceedings of the 6th ACM conference on Embedded network sensor systems, pages 295308. ACM, 2008.

[24] Chih-Wei Lai, Yu-Lung Lo, Jiahn-Piring Yur, and Chin-Ho Chuang. Application of fiber bragg grating level sensor and fabry-perot pressure sensor to simultaneous measurement of liquid level and specific gravity. Sensors Journal, IEEE, 12(4):827-831, 2012.

[25] Edward Canepa, Enas Odat, Ahmad Dehwah, Mustafa Mousa, Jiming Jiang, and Christian Claudel. A sensor network architecture for urban traffic state estimation with mixed Eulerian/Lagrangian sensing based on distributed computing. 27th Conference on Architecture of Computing Systems, 2014.

[26] Jiming Jiang and Christian Claudel. A wireless computational platform for distributed computing based traffic monitoring involving mixed eulerian-lagrangian sensing. In Industrial Embedded Systems (SIES), 2013-8th IEEE International Symposium on, pages 232-239. IEEE, 2013.

[27] Daniele Marioli, Claudio Narduzzi, Carlo Offelli, Dario Petri, Emilio
Sardini, and Andrea Taroni. Digital time-of-flight measurement for ultrasonic sensors. IEEE Transactions on Instrumentation and Measurement, 41(1):93-97, 1992.

[28] Jagdish Chandra Patra, Pramod Kumar Meher, and Goutam Chakraborty. Development of laguerre neural-network-based intelligent sensors for wireless sensor networks. Instrumentation and Measurement, IEEE Transactions on, 60(3):725-734, 2011.

[29] Jagdish Chandra Patra, Alex C Kot, and Ganapati Panda. An intelligent pressure sensor using neural networks. Instrumentation and Measurement, IEEE Transactions on, 49(4):829-834, 2000

[30] Bimal K Bose. Neural network applications in power electronics and motor drives an introduction and perspective. Industrial Electronics, IEEE Transactions on, 54(1):14-33, 2007.

[31] Magali RG Meireles, Paulo EM Almeida, and Marcelo Godoy Simões. A comprehensive review for industrial applicability of artificial neural networks. Industrial Electronics, IEEE Transactions on, 50(3):585-601, 2003.

[32] Franck Betin, Arnaud Sivert, Amine Yazidi, and G-A Capolino. Determination of scaling factors for fuzzy logic control using the slidingmode approach: Application to control of a dc machine drive. Industrial Electronics, IEEE Transactions on, 54(1):296-309, 2007.

[33] Shuzhi Sam Ge and Cong Wang. Adaptive neural control of uncertain mimo nonlinear systems. Neural Networks, IEEE Transactions on, 15(3):674-692, 2004.

[34] Frank L Lewis, Aydin Yesildirek, and Kai Liu. Multilayer neuralnet robot controller with guaranteed tracking performance. Neural Networks, IEEE Transactions on, 7(2):388-399, 1996.

[35] Kurt Hornik, Maxwell Stinchcombe, and Halbert White. Multilayer feedforward networks are universal approximators. Neural networks, 2(5):359-366, 1989.

[36] Christopher M Bishop et al. Pattern recognition and machine learning, volume 1. springer New York, 2006.

[37] Brian D Ripley and Ruth M Ripley. Neural networks as statistical methods in survival analysis. Clinical applications of artificial neural networks, pages 237-255, 2001.

[38] Jorge J Moré. The levenberg-marquardt algorithm: implementation and theory. In Numerical analysis, pages 105-116. Springer, 1978.

[39] Seong Taek Chung, Seung Jean Kim, Jungwon Lee, and John M Cioffi. A game-theoretic approach to power allocation in frequencyselective gaussian interference channels. In in Proc. IEEE International Symposium on Inform. Theory, Pacifico. Citeseer, 2003.

[40] Mark Schmidt. Least squares optimization with 11-norm regularization. CS542B Project Report, 2005.

[41] Biswanath Bhattacharya and Dimitri P Solomatine. Neural networks and $\mathrm{m} 5$ model trees in modelling water level-discharge relationship. Neurocomputing, 63:381-396, 2005.

[42] Victor B Veiga, Quazi K Hassan, and Jianxun He. Development of flow forecasting models in the bow river at calgary, alberta, canada. Water, 7(1):99-115, 2014.

[43] Saudi flash floods, http://www.emirates247.com/news/region / saudi-flash-floods-one-killed-in-makkah-2014-05-10-1.548596.

[44] ARM uvision Keil software, http://www2.keil.com/mdk5/uvision//. 